\title{
Focused Registration of Tracked 2D US to 3D CT Data of the Liver
}

\author{
Janine Olesch ${ }^{1,2,3}$, Bernd Fischer ${ }^{1,2}$ \\ ${ }^{1}$ Institute of Mathematics and Image Computing, University of Lübeck, Germany; \\ ${ }^{2}$ Fraunhofer MEVIS, Project Group Image Registration, Lübeck, Germany; \\ ${ }^{3}$ Graduate School for Computing in Medicine and Life Sciences, Univ. Lübeck, \\ Germany \\ olesch@mic.uni-luebeck.de
}

\begin{abstract}
The paper deals with the registration of pre-operative 3DCT-data to tracked intra-operative 2D-US-slices in the context of liver surgery. To bring such a method to clinical practice, it has to be fast and robust. In order to meet these demanding criteria, we propose two strategies. Instead of applying a time-consuming compounding process to obtain a 3D-US image, we use the 2D-slices directly and thereby drastically reduce the complexity and enhance the robustness of the scheme. Naturally, the surgeon does not need the same high resolution for the whole liver. We make use of this fact by applying a focusing technique to regions of special interest. With this, we reduce the overall amount of data to register significantly without sacrificing the accuracy in the ROIs. In contrast to other attempts, the high resolution result in the ROI is combined in a natural way with a global deformation field to obtain a smooth registration of the whole liver. Overall we arrive at a method with a favorable timing. The proposed algorithm was applied to four different patient data-sets and evaluated with respect to the reached vessel-overlap on validation slices. The obtained results are very convincing and will help to bring non-linear registration techniques to the operation theater.
\end{abstract}

\section{Introduction}

The best treatment for tumors in the liver is still their resection. This is a tricky intervention, as one would like to remove all tumorous tissue and to preserve as much healthy liver as possible. To this end planning-data is acquired and processed, which ideally provides an optimal resection plan. As the liver is deformed during the intervention, intra-operative navigation is needed to guide the surgeon with respect to the planning-data. To enable the navigation during the intervention, ultrasound (US) is often the method of choice. It is the purpose of this paper to come up with a reliable and fast scheme which aligns the pre-processed $3 \mathrm{D}$ planning-data $(\mathrm{CT})$ to the intra-operative situation, given the information by the tracked 2D-US-slices. Instead of determining a non-linear transformation for the whole liver, we propose a different strategy: In the first step we perform 
a fast rigid pre-alignment based on the full CT-volume. The second step is then used to improve this result non-linearly in a region of interest (ROI). As the non-linear step is computationally more expensive as opposed to the rigid one and the surgeon is not interested in the same resolution throughout the whole liver, it makes very much sense to focus on a ROI. To achieve nevertheless a transformation for the full volume, we apply a technique developed in [1], where the authors introduce the idea of focused registration. Figure 1 (a) visualizes pre-processed 3D planning-data superimposed by the proposed resection plane, the ROI (b), (c), and some of the US-slices (d). It should be noted, that the ROI is automatically derived from the location of the resection plane and the associated US-slices.

\section{Materials and Methods}

We start by briefly describing the different steps of our proposed method. First we select $j_{\text {rigid }}$ slices from the US-data based on an entropy measure. Next, the rigid registration process with respect to these slices and the CT-data takes place. Then, based on the resection plane, we identify a region of interest $\Omega_{\mathrm{ROI}}$ in the CT-volume. Subsequently, the US-slices within the ROI are determined. For the non-linear step, a subset of $j_{\mathrm{nl}}$ slices is selected. Naturally, the non-linear step makes use of the rigid result on $\mathcal{T}_{\text {ROI }}$ and is performed solely based on the selected US-slices. Finally, the result $y_{\text {opt }}$ of the non-linear step is combined with the result of the rigid step to gain the deformation for the full CT-volume.

The registration-steps described above are based on the idea of volume-toslice registration, which was introduced for rigid registration by Penney et al. [2] and for non-linear registration by Heldmann and Papenberg [3]. In the following we describe the specialized non-linear registration problem that will be solved using the discretize-then-optimize approach in our proposed algorithm. In the first step we apply the rigid volume-to-slice registration, as described in [4]. The general version of the non-linear registration problem reads as follows: given a template $\mathcal{T}$ and a reference $\mathcal{R}$, where $\mathcal{T}, \mathcal{R}: \Omega \subset \mathbb{R}^{3} \rightarrow \mathbb{R}$, find a transformation $y: \mathbb{R}^{3} \rightarrow \mathbb{R}^{3}$ such that

$$
\mathcal{J}(y)=\mathcal{D}(R, T ; y)+\alpha \mathcal{S}(y)=\min !
$$

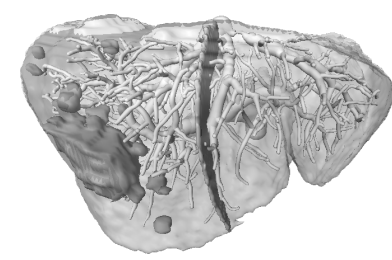

(a)

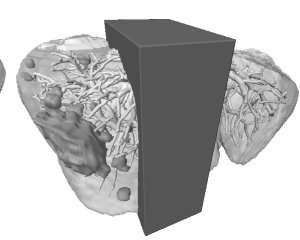

(b)

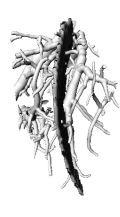

(c)

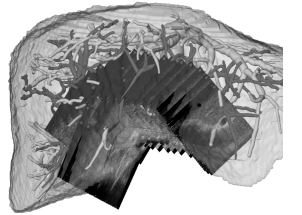

(d)

Fig. 1. CT planning-data including the vessel-system (a), the lesions and a proposed resection plane; bounding box covering the ROI (b); stripped version of ROI (c); liver and some of the tracked 2D-US-slices (d). 
where $\mathcal{D}$ denotes a distance measure, $\mathcal{S}$ a regularizer, and $\alpha \in \mathbb{R}^{+}$a regularization parameter.

The present registration problem is originally a multi-modal registration problem (US-CT). To circumvent this, we make use of the fact that the vessel systems are of foremost interest for the surgeon. More precisely, we only register the segmented vessel systems $\mathcal{T}^{V}$ and $\mathcal{R}^{V}$. Note, that the CT-data is already segmented in the planning step. Consequently, we are able to treat the former multi-modal data by the cheap sum of squared differences (SSD) distance measure. The distance measure, which is evaluated only on the known $j_{\mathrm{nl}}$ slices $M_{j}^{\mathrm{nl}}, j=1, \ldots, j_{\mathrm{nl}}$ reads

$$
\mathcal{D}^{\text {slice }}(y)=\sum_{j=1}^{j_{\mathrm{nl}}} \int_{M_{j}^{\mathrm{nl}}}\left(\mathcal{T}^{V}(y(x))-\mathcal{R}^{V}(x)\right)^{2} d s(x)
$$

where $d s(x)$ denotes the two-dimensional surface measure.

To arrive at smooth deformations and to regularize the ill-posed problem for the non-linear step, a regularization term $\mathcal{S}$ is needed. For the special case of volume-to-slice registration we need a high order regularizer in order to assure a smooth deformation field $y$ in between the US-slices [3]. To this end, $\mathcal{S}$ is chosen as the second order curvature regularizer [5]

$$
\mathcal{S}^{\text {curv }}(y)=\sum_{\ell=1}^{3} \int_{\Omega_{\mathrm{ROI}}}\left|\Delta y_{\ell}\right|^{2} d x
$$

In contrast to the distance measure, the regularizer works on the full deformation field ( $\Omega_{\mathrm{ROI}}$ instead of $\left.M_{j}^{\mathrm{nl}}\right)$.

The discretize-then-optimize framework makes it quite straightforward to apply the idea of focused registration to our scheme. To calculate a result, which covers the full CT-volume, we apply Dirichlet zero boundary conditions to the regularizer in the focused non-linear step. With this we are able to combine the rigid and the non-linear result in an interpolation-step to an overall deformationfield.

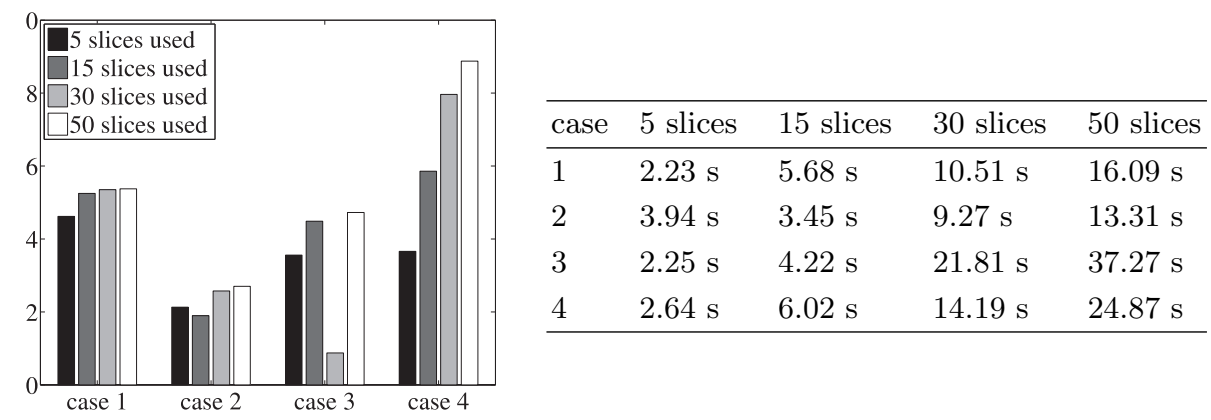

Fig. 2. Factors of improvement of the rigid registration step in relation to the vesseloverlap before rigid registration (left) and run-times (right), for all four different cases. 
In the beginning of this section, we mentioned two steps, where we are obliged to select slices from the US-data. Those slices, in conjunction with the regularizer, are supposed to be sufficient to guide the registration. It is obviously important that the selected slices are distributed throughout the volume to be registered and that they contain meaningful information. We therefore apply a strategy proposed by Wein et.al [6], that is, we partition the original slices into several groups and choose slices based on their entropy with these groups. Only the selected slices are then used to calculate the deformation. We tested different numbers of slices and evaluated the outcome of the methods based on these numbers. To further speed up the computation and to avoid local minima we apply a multi-level strategy in the rigid as well as in the non-linear registration step. We start on a broad resolution of the chosen slices and refine it, until a user prescribed finest resolution. Simultaneously we apply a multi-scale approach to the CT-volume that starts with the main vessels and includes stepwise finer vessels using morphological operations [7]. To solve the final registration problem we apply the discretize-then-optimize strategy [8] by evoking the Gauss-Newton method.

\section{Results}

We tested the proposed algorithm on four different cases. Note that all runtime results were obtained using MATLAB 2010b code, on a $2 \times 2.8 \mathrm{GHz}$ Quad-Core Intel Xeon Mac Pro, which was not optimized for run-time. To evaluate the algorithm, different configurations of the parameters were chosen. For the multi-level setup-up we chose two rigid steps and two non-linear steps in all tests. First we evaluated the influence of the number of slices used in the rigid registration step. The validation subset of slices is fixed throughout all our validation steps to make the results comparable. The left part of Figure 2 visualizes the factors of improvement in relation to the vessel-overlap before rigid registration. This together with the run-time table suggests that a choice

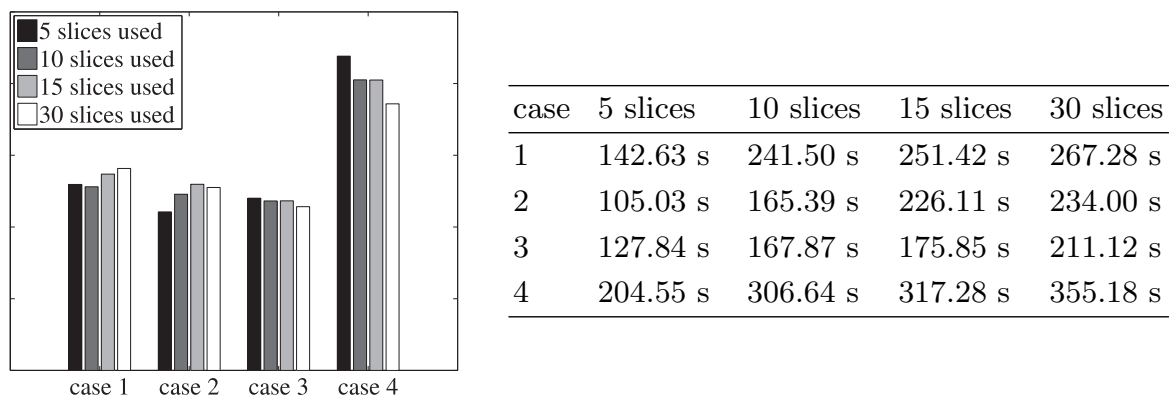

Fig. 3. Factors of improvement of non-linear registration in relation to the vesseloverlap after rigid registration (left) and run-times (right), for all four different cases. 
of 15 slices seems to be a reasonable compromise between favorable run-time and accuracy for the rigid step. The non-linear steps are performed in the region of interest solely and started from the rigid results based on 15 slices. We tested the non-linear step also on different numbers of slices. Knowing that the number of slices will have direct impact on the run-times we chose $\left(M_{j}^{\mathrm{nl}}, j=5,10,15,30\right)$. For non-linear registration the choice of $\alpha$ is always a crucial step, we tested different possibilities. In our tests $\alpha=0.5$ turned out to be the best choice in terms of improvement of the results with respect to run-times and regularity of the resulting grid. Figure 3 indicates for the non-linear step in the regions of interest, choices of $M_{j}^{\mathrm{nl}}, j=5,10$ seem to be equally reasonable, resulting in an improvement up to factor of two.

\section{Discussion}

For the first time, the focused registration methodology is combined with rigid and non-linear volume to slice registration techniques. We tested the method on four clinical data-sets and observe very promising results both in terms of timing and accuracy. Future plans include the evaluation on more clinical data-sets and the porting of the code to a run-time optimized environment.

\section{References}

1. Papenberg N, Modersitzki J, Fischer B. Registrierung im Fokus. In: Proc BVM; 2008. p. 138-42.

2. Penney GP, Blackall JM, Hamady MS, et al. Registration of freehand 3D ultrasound and magnetic resonance liver images. Med Image Anal. 2004;8(1):81-91.

3. Heldmann S, Papenberg N. A variational approach for volume-to-slice registration. Lect Notes Computer Sci. 2009;4485:624-35.

4. Heldmann S, Beuthien B, Olesch J, et al. Improved minimal-invasive laproscopic liver surgery by regstration of 3D CT and 2D ultrasound slices. Proc BMT. 2010.

5. Fischer B, Modersitzki J. Fast curvature-based registration of MR mammography images. In: Proc BVM; 2002. p. 139-42.

6. Wein W, Brunke S, Khamene A, et al. Automatic CT-ultrasound registration for diagnostic imaging and image-guided intervention. Med Image Anal. 2008;12:57785 .

7. Heldmann S, Papenberg N. A scale-space approach for image registration of vessel structures. In: Proc BVM; 2009. p. 137-41.

8. Modersitzki J. FAIR: Flexible Algorithms for Image Registration. Philadelphia: SIAM; 2009. 